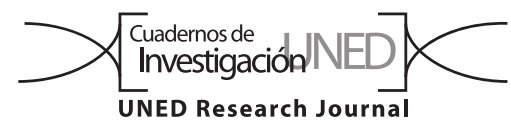

\title{
Ciclo reproductivo de la ardilla Sciurus variegatoides (Rodentia: Sciuridae) en Costa Rica
}

\author{
Javier Monge \\ Universidad de Costa Rica, Escuela de Agronomía, Centro de Investigación en Protección de Cultivos, Facultad de Ciencias Agroalimentarias, \\ Instituto de Investigaciones Agrícolas; javier.monge@ucr.ac.cr
}

Recibido 01-VIII-2018 • Corregido 19-X-2018 • Aceptado 24-X-2018

\begin{abstract}
Reproductive cycle of the squirrel Sciurus variegatoides (Rodentia: Sciuridae) in Costa Rica". Introduction: the squirrel Sciurus variegatoides is one of the most abundant species of squirrels in Central America, yet little is known about its reproduction. Objective: to determine the reproductive cycle of $S$. variegatoides in an agricultural area of Costa Rica. Methods: monthly (Dec. 1986 to Jan. 1988), I collected 10 animals at Hacienda Curú, Paquera, Costa Rica. I used scrotum presence, testicle size and length of the uterine horns to assess maturity. The reproductive cycle was based on pregnancy, lactation, courtship, nest construction, and proportion of subadults. Results: I collected a total 135 squirrels; sex ratio was 1,7 males per female; and $22 \%$ were subadults, both for males and females. I captured the pregnant females from December to June and represented $42 \%$ of the adult females. February and April were marked by courtship and an increase in the construction of nests. I collected lactating females from June to September, when I captured more subadults. Conclusion: There was no evidence of reproductive activity in October and November.
\end{abstract}

Key words: squirrels, reproduction, population structure, Sciurus variegatoides, Costa Rica.
RESUMEN: Introducción: la ardilla Sciurus variegatoides es una de las especies más abundantes de ardilla en América Central, sin embargo, poco se sabe sobre su reproducción. Objetivo: determinar el ciclo reproductivo de $S$. variegatoides en un área agrícola de Costa Rica. Métodos: mensualmente (diciembre de 1986 a enero de 1988) recolecté 10 animales en Hacienda Curú, Paquera, Costa Rica. Usé la presencia de escroto, el tamaño de los testículos y la longitud de los cuernos uterinos para evaluar la madurez. El ciclo reproductivo se basó en la preñez, la lactancia, el cortejo, la construcción de nidos y la proporción de subadultos. Resultados: recogí un total de 135 ardillas, la proporción de sexos fue de 1,7 machos por cada hembra y el $22 \%$ fueron subadultos (ambos sexos). Capturé las hembras embarazadas de diciembre a junio y representaron el $42 \%$ de las hembras adultas. Febrero y abril estuvieron marcados por cortejo y un aumento en la construcción de nidos. Recolecté las hembras lactantes de junio a setiembre, cuando capturé más subadultos. Conclusión: no hubo evidencia de actividad reproductiva en octubre y noviembre.

Palabras clave: ardillas, reproducción, estructura poblacional, Sciurus variegatoides, Costa Rica.
La ardilla Sciurus variegatoides Ogilby, 1839 es una de las nueve especies de la familia Sciuridae presentes en América Central (Thorington \& Hoffmann, 2005), de la cual se dispone de relativa poca información, limitándose una descripción de sus subespecies (Harris, 1937; McPherson, 1971), su alimentación (Glanz, 1984; Monge \& Hilje, 2006; Henn, McCoy, \& Vaughan, 2014) y algunos datos de estudios no enfocados a la especie o bien de observaciones aisladas (Boucher, 1981; Fleming, 1970; Janzen, 1971, 1972, 1982a, 1982b; Rose et al., 2003; Schemske, 1980). Otros aspectos de su historia natural han sido resumidos por Best (1995). A su vez, McPherson (1985) la incluye dentro de un análisis biogeográfico de roedores en Costa Rica, así como Hayssen (2008) en un estudio de patrones de largo de cuerpo y cola y peso de ardillas.
Esta escasez de información contrasta con el hecho de ser una especie abundante y localmente común (Thorington, Koprowski, Steele, \& Whatton, 2012), y que según McPherson (1985) en Costa Rica se conocía de más lugares que en cualquier otro roedor, cuyas poblaciones alcanzan densidades importantes como para ser consideradas plagas agrícolas (Hilje \& Monge, 1988; Monge, 2009). A su vez, al ser un animal diurno (Reid, 2009) y de relativa facilidad para ser observado (Somarriba, et al., 2003) sería esperable que se dispusiera de información documentada, aunque fuera de observaciones asiladas.

En cuanto a la reproducción de la especie se hace referencia a que la crianza ocurre en abril y mayo (Best, 1995) y que el tamaño de la camada es probablemente de cuatro a seis jóvenes (Goodwin, 1946) o de tres a cinco crías (Méndez, 1993). 
El propósito de este estudio fue determinar el ciclo reproductivo de $S$. variegatoides en un área agrícola en Costa Rica.

\section{MATERIALES Y MÉTODOS}

El estudio se efectuó en la Hacienda Curú, en el cantón Paquera, provincia de Puntarenas, ubicada en la región suroeste de la Península de Nicoya, Costa Rica $\left(9^{\circ} 47^{\prime} \mathrm{N} \& 84^{\circ} 56^{\prime} \mathrm{W}\right)$. La precipitación media anual es de $1957,43 \mathrm{~mm}$, con una época seca (precipitación mensual inferior a $50 \mathrm{~mm}$ ) que abarca desde diciembre hasta abril, y una época lluviosa de mayo a noviembre, siendo los meses de setiembre y octubre los más lluviosos con precipitaciones mensuales superiores a $300 \mathrm{~mm}$. La temperatura promedio mensual varía entre $25-30^{\circ} \mathrm{C}$, según datos vigentes en el momento en que se realizó el estudio. En el sitio se encuentran las zonas de vida bosque húmedo premontano transición a basal y bosque seco tropical (Tosi, 1969).

El estudio se desarrolló desde diciembre de 1986 hasta enero de 1988. Cada mes se colectó diez individuos, excepto los dos primeros meses que se colectó siete individuos, y 11 en julio, los cuales fueron sacrificados con rifles calibre 22, como parte del control dada su condición dañina para la plantación de coco. Todos los individuos fueron colectados en áreas agrícolas, o bien de potreros arbolados o cercas vivas que bordeaban áreas de potrero. No se incluyeron individuos que se encontraran en bosques. Parte de la muestra colectada se encuentra depositada en la colección del Departamento de Historia Natural del Museo Nacional de Costa Rica (MNCR 763774 y MNCR 776-778).

Cada espécimen fue medido (largo total y de cola) y pesado, además de determinado su sexo. En los machos, se observó el estado de los genitales, considerándose la presencia o ausencia de escroto, asumiéndose su ausencia como indicativo de inmadurez sexual o subadulto. De las hembras, se observó el desarrollo de las mamas para determinar el estado de lactancia.

Posteriormente, se hizo una incisión abdominal para extraer el aparato reproductor. En el caso de las hembras se observó el estado de preñez por la presencia de embriones, los cuales fueron contabilizados, y también se midió la longitud de los cuernos uterinos. Para los machos, se tomaron medidas de longitud y amplitud de los testículos, valores con los que se estimó un tamaño promedio de testículos, obtenido de la media de las cuatro mediciones, el cual fue utilizado como criterio de edad complementario a la presencia o ausencia de escroto. También se observó para el momento de la captura, si el individuo se encontraba en actividades de cortejo, la cual al igual de otras especies de ardillas arborícolas se caracteriza en la persecución activa de varios machos a una hembra (Heaney, 1991). Esta información permitió definir preliminarmente criterios de edad, los cuales no están definidos para esta especie.

Ética, conflicto de intereses y declaración de financiamiento: el autor declara haber cumplido con todos los requisitos éticos y legales pertinentes, tanto durante el estudio como en el manuscrito; que no hay conflictos de interés de ningún tipo, y que todas las fuentes financieras se detallan plena y claramente en la sección de agradecimientos. Asimismo, está de acuerdo con la versión editada final del documento. El respectivo documento legal firmado se encuentra en los archivos de la revista.

\section{RESULTADOS}

Se capturó 135 individuos de Sciurus variegatoides atrirufus, de los cuales 85 fueron machos y 50 hembras (Cuadro 1), lo que implica una proporción de 1,7:1 (machos:hembras). La longitud corporal (largo total-largo de cola) osciló entre 207 y $319 \mathrm{~mm}(269 \pm 16,9 \mathrm{~mm})$, siendo para los machos entre 207 y $319 \mathrm{~mm}(270,7 \pm 17,0 \mathrm{~mm})$, y para las hembras entre 216 y $308 \mathrm{~mm}(267,8 \pm 16,8 \mathrm{~mm})$. La longitud de cola osciló entre 202 y $294 \mathrm{~mm}$ $(260,9 \pm 78,6 \mathrm{~mm})$, para los machos de 205 y $294 \mathrm{~mm}$ $(260,0 \pm 77,9 \mathrm{~mm})$ y para las hembras entre 230 y $281 \mathrm{~mm}$ $(262,5 \pm 80,4 \mathrm{~mm})$. En cuanto al peso, osciló entre 185 y $670 \mathrm{~g}(521,8 \pm 89,3 \mathrm{~g})$, cuyos valores para los machos fueron entre 185 y $625 \mathrm{~g}(518,4 \pm 84,6 \mathrm{~g})$ y para las hembras de 275 y $670 \mathrm{~g}(527,4 \pm 97,5 \mathrm{~g})$. En la Fig. 1 se observa la relación entre la longitud y peso corporal según categorías de sexo y edad.

Clasificación por edad: Los individuos de S. variegatoides pueden clasificarse en tres categorías de edad: juveniles, subadultos y adultos. Por el comportamiento de las ardillas arborícolas, los juveniles se mantienen en los nidos o en sus alrededores, por lo que en este caso no formaron parte de la muestra, dada la forma de captura utilizada. En el caso de los machos, se consideró como subadultos a aquellos individuos carentes de escroto, los que a su vez tuvieron un tamaño promedio de testículos inferior a $8,5 \mathrm{~mm}$, lo cual representa un $48 \%$ del tamaño testicular promedio de los individuos clasificados como adultos. Por su parte, como macho adulto se clasificó a aquellos individuos con escroto, aun cuando su escroto estuviera en estado desarrollo y tuviera alguna porción 
CUADRO 1

Capturas mensuales de Sciurus variegatoides

\begin{tabular}{|c|c|c|c|c|c|c|c|c|}
\hline \multirow{2}{*}{ Mes } & \multicolumn{2}{|c|}{ Subadultos } & \multicolumn{2}{|c|}{ Adultos } & \multirow{2}{*}{$\begin{array}{c}\text { Machos en } \\
\text { cortejo }\end{array}$} & \multirow{2}{*}{$\begin{array}{l}\text { Hembras en } \\
\text { cortejo }\end{array}$} & \multirow{2}{*}{$\begin{array}{l}\text { Hembras } \\
\text { preñadas }\end{array}$} & \multirow{2}{*}{$\begin{array}{l}\text { Hembras } \\
\text { lactando }\end{array}$} \\
\hline & Machos & Hembras & Machos & Hembras & & & & \\
\hline Dic. & 1 & 0 & 4 & 2 & 0 & 0 & 1 & 0 \\
\hline Ene. & 2 & 0 & 5 & 0 & 0 & 0 & 0 & 0 \\
\hline Feb. & 0 & 0 & 8 & 2 & 3 & 1 & 1 & 0 \\
\hline Mar. & 0 & 0 & 9 & 1 & 0 & 0 & 1 & 0 \\
\hline Abr. & 1 & 0 & 6 & 3 & 4 & 0 & 1 & 0 \\
\hline May. & 1 & 1 & 5 & 3 & 0 & 0 & 1 & 0 \\
\hline Jun. & 1 & 1 & 2 & 6 & 0 & 0 & 2 & 3 \\
\hline Jul. & 4 & 1 & 3 & 3 & 0 & 0 & 0 & 1 \\
\hline Ago. & 1 & 1 & 5 & 3 & 0 & 0 & 0 & 1 \\
\hline Set. & 3 & 2 & 2 & 3 & 0 & 0 & 0 & 2 \\
\hline Oct. & 1 & 2 & 4 & 3 & 0 & 0 & 0 & 0 \\
\hline Nov & 1 & 2 & 5 & 2 & 0 & 0 & 0 & 0 \\
\hline Dic. & 1 & 1 & 4 & 4 & 0 & 0 & 0 & 0 \\
\hline Ene. & 2 & 0 & 4 & 4 & 0 & 0 & 2 & 0 \\
\hline Total & 19 & 11 & 66 & 39 & 7 & 1 & 9 & 7 \\
\hline
\end{tabular}

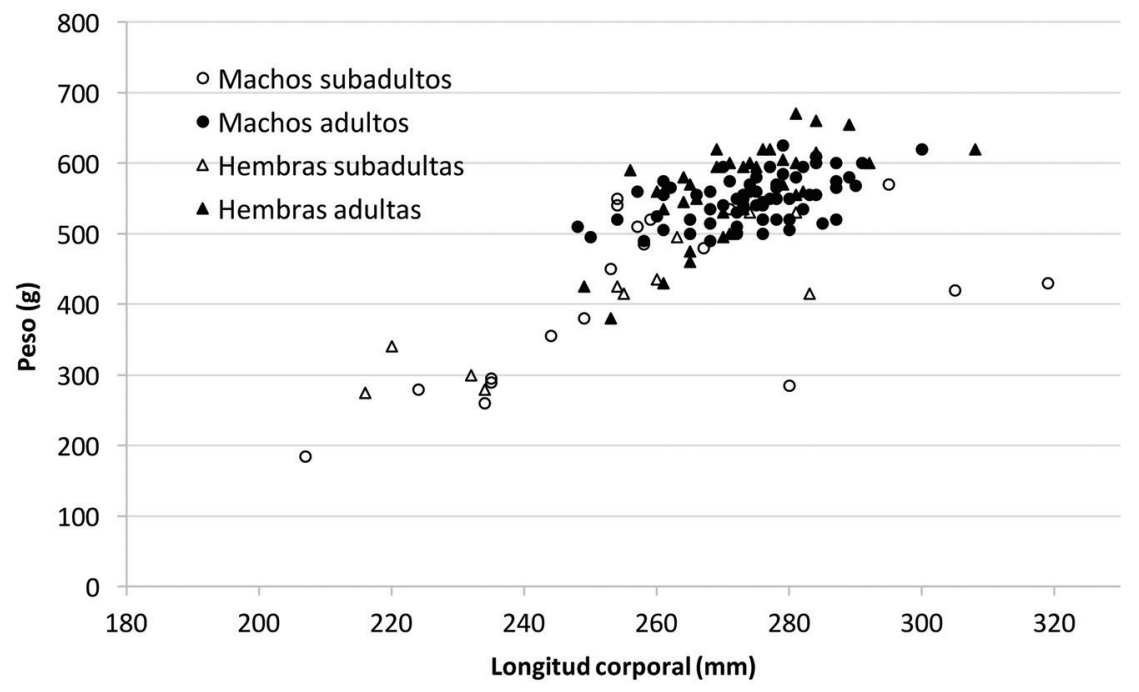

Fig. 1. Relación entre longitud y peso corporal de S. variegatoides.

con pelo, cuyo tamaño promedio testicular osciló entre 10,64 y $20,80 \mathrm{~mm}(17,76 \pm 2,20 \mathrm{~mm})$. Para las hembras se consideró subadultas a aquellos individuos con sistema reproductivo pequeño, es decir con longitud de cuerno uterino inferior a $20 \mathrm{~mm}$, cuyo tamaño representan un $61 \%$ del promedio del tamaño de cuernos de hembras preñadas, lactando o en cortejo, que osciló entre 21 y $62 \mathrm{~mm}(32,9 \pm 9,8 \mathrm{~mm})$.

Por los criterios anteriores, se tiene que de los 85 machos, 19 individuos fueron clasificados como subadultos y 66 adultos, mientras que de las 50 hembras capturadas 11 se clasifican como subadultas y 39 como adultas. Para ambas clases de edad la proporción de sexos fue de 1,7:1 (machos:hembras). El criterio de largo y peso corporal para clasificar a los individuos por edad no se consideró útil, ya que individuos pertenecientes a ambas clases de edad, se traslapan en su tamaño y masa corporal. Sin embargo, hay una proporción de individuos que no alcanzan el ámbito de longitud y peso observado para individuos clasificados como adultos, por lo que podría 
CUADRO 2

Longitud y peso corporal de S. variegatoides según sexo y edad

\begin{tabular}{|c|c|c|c|c|}
\hline & \multicolumn{2}{|c|}{ Subadultos } & \multicolumn{2}{|c|}{ Adultos } \\
\hline & Longitud (mm) & Peso (g) & Longitud (mm) & Peso (g) \\
\hline \multicolumn{5}{|l|}{ Ambos sexos } \\
\hline Ámbito & 207-319 & $185-570$ & $248-308$ & $380-670$ \\
\hline Promedio \pm D.E & $255,7 \pm 26,0$ & $408,7 \pm 108,1$ & $273,1 \pm 10,5$ & $554,1 \pm 47,8$ \\
\hline \multicolumn{5}{|l|}{ Machos } \\
\hline Ámbito & $207-319$ & $185-570$ & $248-300$ & $490-625$ \\
\hline Promedio \pm D.E. & $257,9 \pm 27,6$ & $411,6 \pm 117,8$ & $274,4 \pm 10,0$ & $549,2 \pm 33,2$ \\
\hline \multicolumn{5}{|l|}{ Hembras } \\
\hline Ámbito & $216-283$ & $275-530$ & $249-308$ & $380-670$ \\
\hline Promedio \pm D.E. & $252,0 \pm 23,5$ & $403,6 \pm 94,1$ & $272,2 \pm 11,2$ & $562,3 \pm 65,2$ \\
\hline
\end{tabular}

considerar que la longitud corporal inferior a $240 \mathrm{~mm}$ y peso inferior a $340 \mathrm{~g}$ corresponde a subadultos, lo cual sería aplicable para machos y hembras. Al considerarse estas categorías de edad, se tiene que la longitud corporal promedio de los adultos de $273,1 \pm 10,5 \mathrm{~mm}$ y de peso de $554,1 \pm 47,8 \mathrm{~g}$, mientras que para el grupo de los subadultos fue de $255,7 \pm 26,0 \mathrm{~mm}$ y de $408,7 \pm 108,1 \mathrm{~g}$, respectivamente (Cuadro 2).

Ciclo reproductivo: La actividad reproductiva de las hembras se desarrolló entre diciembre y junio, periodo en que algunas hembras se encontraban en estado de preñez, lo que corresponde a la época seca y principios de la lluviosa, periodo en que se observó mayor construcción de nidos. Las hembras preñadas de las muestras de ese periodo representan el $43 \%$ de las hembras adultas capturadas. El tamaño promedio de las camadas fue de 1,67+0,82 embriones por hembra, y variaron entre uno y tres. La camada mayor fue recolectada en mayo.

Durante los meses de febrero (tres veces) y en abril (una vez) se observaron actividades de cortejo, las cuales ocurrieron en horas de la mañana (06-08h). Los grupos en cortejo estuvieron constituidos por una hembra y de cuatro a seis machos. La actividad se caracterizó por una persecución activa de los machos a la hembra en un área relativamente pequeña. En una ocasión se observó que se ocupó un área de aproximadamente $900 \mathrm{~m}^{2}$ en un área plantada de cocoteros, que contenía menos de diez palmas.

El ciclo reproductivo lo complementa la lactancia, cuya colecta de hembras con muestras de encontrarse en periodo de lactancia ocurrió durante los meses de junio a setiembre, de las cuales dos hembras lactantes se capturaron junto con individuos clasificados como subadultos en la muestra de setiembre.

Los individuos clasificados como subadultos se capturaron casi todo el año, excepto en febrero y marzo, y con mayor frecuencia entre julio y noviembre, periodo durante el cual la proporción de subadultos osciló entre $45-50 \%$ de la muestra de julio y setiembre y un $30 \%$ en octubre y noviembre (Fig. 2). Sin embargo, por el tamaño y peso corporal de los subadultos no sugieren tener la misma edad, por lo que al considerar los de menor tamaño y peso, se tiene que el individuo más pequeño se capturó en diciembre, y los otros nueve individuos en orden de tamaño y peso se concentraron entre junio y setiembre, periodo que coincide con el periodo de mayor captura de hembras lactantes.

Dado el comportamiento de crianza de las ardillas de esta especie, la construcción de nidos es parte del proceso reproductivo. La construcción de nidos fue más evidente en mayo, cuando se lograron observar varios nidos nuevos por árbol y mayor cantidad de árboles con nidos. En diciembre se observó una ardilla construyendo un nido en un árbol de mango (Mangifera indica), para lo cual utilizó hojas del mismo árbol. Ella usó hojas verdes para construir los nidos, y fueron transportadas una por una, o en pequeñas ramas. Los árboles utilizados para la instalación de los nidos eran frondosos, con muchas ramificaciones, y los nidos fueron establecidos a una altura superior a $15 \mathrm{~m}$.

En resumen, se puede considerar que la preñez ocurre entre diciembre y junio, con una mayor actividad de cortejo entre febrero y abril, seguido por un incremento de construcción de nidos en mayo y posteriormente un periodo de lactancia entre junio y setiembre. 


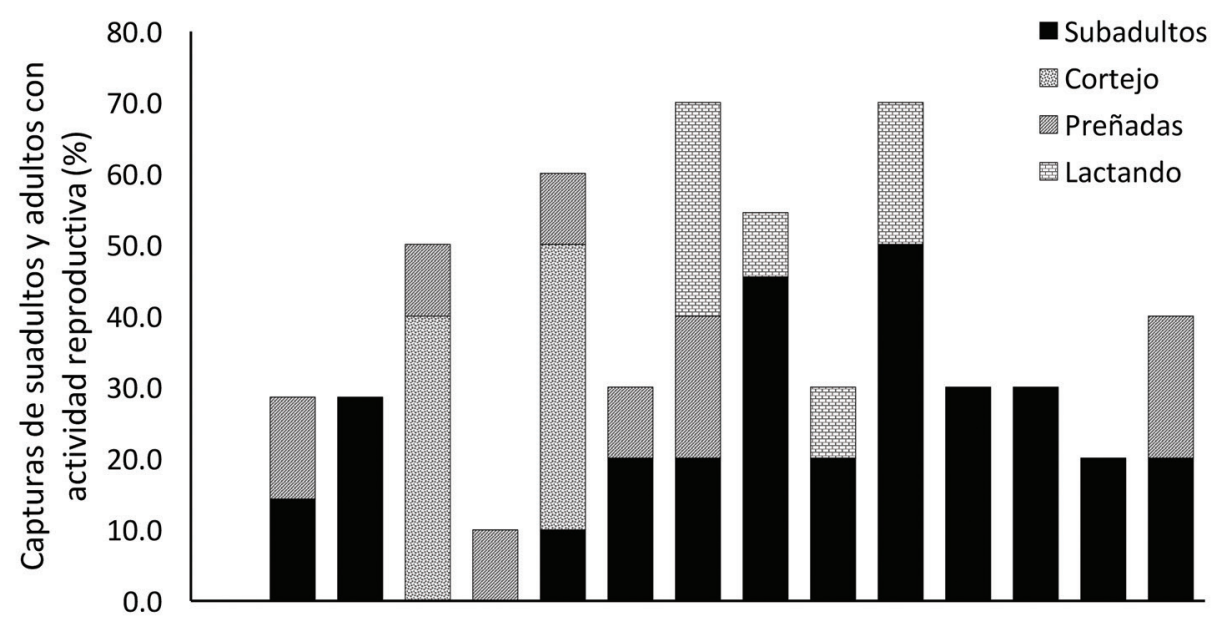

Dic. Ene. Feb. Mar. Abr. May. Jun. Jul. Ago. Set. Oct. Nov Dic. Ene.

Meses

Fig. 2. Subadultos y adultos en actividad reproductiva de S. variegatoides.

\section{DISCUSIÓN}

La población de estudio corresponde a la subespecie S. v. atrirufus cuyas dimensiones corporales oscilaron entre 207 y $319 \mathrm{~mm}$ para la longitud corporal, incluyendo individuos de ambos sexos y edad, el largo de cola entre $202-294 \mathrm{~mm}$ y el peso entre $185-670 \mathrm{~g}$, las cuales coinciden parcialmente con reportes previos para la especie, con longitud corporal entre 240-300 (Reid, 2009), largo de cola de 239-320mm (Reid, 2009; Best, 1995) y peso de 447-909g (Reid, 2009). Las diferencias pueden deberse a que otras referencias incluyen individuos de otras subespecies, las que eventualmente puedan diferir en tamaño y peso. La diferencia mayor se presenta en el peso, dado que el valor máximo de la muestra de estudio representa un $74 \%$ del reportado para la especie. Los valores para machos y hembras no evidenciaron un dimorfismo sexual.

En cuanto a las dimensiones corporales, dado que los subadultos se encuentran en una etapa de crecimiento, los individuos clasificados dentro de este grupo presentan mayores diferencias entre sí, con respecto a lo observado para los adultos. En este caso, el peso corporal fue el que mostró las mayores variaciones, evidenciado por un coeficiente de variación de $28,6 \%$ y $23,3 \%$ para machos y hembras, respectivamente, mientras que para la longitud corporal fue de $10,7 \%$ y $9,3 \%$. Por su parte, para los adultos el coeficiente de variación de la longitud corporal fue de $3,64 \%$ y $4,11 \%$ para machos y hembras, respectivamente, mientras en cuanto al peso fue de $6,05 \%$ para los machos y de $11,60 \%$ para las hembras. Este valor de coeficiente de variación del peso corporal de las hembras puede estar influenciado por las diferencias de peso de las hembras preñadas, ya que las hembras adultas no preñadas tuvieron un peso promedio de $554,8 \pm 71,7 \mathrm{~g}$, mientras que en las hembras preñadas fue de $587,2 \pm 24,6 \mathrm{~g}$. El criterio complementario de peso inferior a $340 \mathrm{~g}$, utilizado para clasificar a los subadultos, coincide con el peso de individuos de Sciurus granatensis clasificados como jóvenes antes del destete, con pesos de 270 y $280 \mathrm{~g}$ y un macho joven con peso de $340 \mathrm{~g}$ (Heaney \& Thorington, 1978) y es similar al valor de $325 \mathrm{~g}$ usado como límite para clasificar a individuos juveniles de Sciurus aureogaster (Brown \& McGuire, 1975).

En cuanto a la estructura poblacional, la proporción de sexos sugiere una mayor presencia de machos, lo cual puede responder a una mayor actividad y por ende mayor probabilidad de ser parte de la muestra. A su vez, la proporción observada puede estar influenciada por los grupos de cortejo que están constituidos por varios machos y solo una hembra, así que al incorporar uno de estos grupos a la muestra es de esperar una mayor representación de machos. En caso de extraer de la clase de adultos a los individuos que formaban parte de grupos de cortejo, la proporción de sexos sería de 1,5:1, lo cual coincide con lo encontrado para S. aureogaster (Brown \& McGuire, 1975). Sin embargo, la misma proporción de 1,7:1 (machos y hembras) fue observada en la clase de subadultos, los cuales no forman parte de los grupos de cortejo, por lo que una mayor proporción de machos puede deberse a una compensación ante una eventual mayor mortalidad de machos o a una estructura poblacional sesgada hacia una mayor proporción de machos. 
Por su parte, Sciurus granatensis mostró una igualdad en la proporción de sexos (Heaney \& Thorington, 1978).

En cuanto a la proporción de individuos según la edad, los subadultos representan un $22 \%$ de la población, proporción observada tanto para machos como para hembras. Esta representación de subadultos es similar a la proporción más alta mostrada por S. aureogaster que fue de $24,4 \%$ y la inferior de $8,8 \%$ (Brown \& McGuire, 1975), pero es inferior a la observada para S. granatensis, que osciló entre $28-56 \%$, con un promedio de $42 \%$ del total de la población (Heaney \& Thorington, 1978).

La colecta de hembras preñadas indica que durante los meses de diciembre a junio es un período de actividad reproductiva, el cual corresponde a la época seca en el sitio de estudio. Este período de presencia de hembras preñadas coincide con lo observado para S. granatesis (Heaney \& Thorington, 1978), periodo en que ambas especies y sitios de estudio muestran más actividades de cortejo, caracterizadas por la persecución de varios machos a una hembra (Glanz, Thorington, GiacaloneMadden, \& Heaney, 1990). El tamaño de las camadas osciló entre uno y tres, con un promedio de 1,67, similar a lo observado por Heaney y Thorington (1978) que fue de dos crías y Glanz et al. (1990) de 1,9 para S. granatensis. A su vez, Jones, Genoways y Lawlor (1974) reportaron tamaños de camadas de entre dos y tres y Birnes, Bowles, Timm y Williams (1974) de tres crías para Sciurus yucatensis, mientras que Reid (2009) de entre dos y cuatro para $S$. aureogaster y de entre dos y tres para Sciurus richmondi.

La observación de actividades de cortejo en este estudio se logró entre febrero y abril, es de esperar que sea más amplio, dada la presencia de hembras preñadas en diciembre, similar a lo observado por Glanz et al. (1990) para S. granatensis. El periodo de captura de hembras lactantes se desarrolló entre junio y setiembre, similar a S. granatensis, cuyas hembras lactantes de capturaron entre mayo y junio. En este período la proporción de subadultos por muestra mensual se incrementó, principalmente en julio y setiembre. La proporción de subadultos ha sido utilizado como criterio de reproducción de $S$. granatensis (Giacalone, Glanz, \& Leigh, 1990). La construcción de nidos fue más evidente en mayo, aunque también se observó en diciembre, lo cual es indicativo de la actividad reproductiva. Este comportamiento de construcción de nidos también ha sido observado en $S$. aureogaster (Brown \& McGuire, 1975; Reid, 2009; RamosLara \& Cervantes, 2011) y en S. deppei y S. yucatensis (Reid, 2009). A partir de octubre no se capturó hembras en actividad reproductiva, lo que también concuerda con lo observado en S. granatensis, época de mayor precipitación en ambos sitios de estudio.
En términos generales, se observa que el ciclo reproductivo de $S$. variegatoides es similar a otras especies de Sciurus de la región, en cuanto a épocas de actividad reproductiva. De igual manera, se evidencia comportamientos similares en cuanto a actividades de cortejo y construcción de nidos. Por lo tanto, se puede recurrir a metodologías usadas para el estudio de otras especies para conocer el ciclo reproductivo de la especie en otras zonas y de otras subespecies. Estas similitudes entre especies permitirían estudiar otros aspectos de la historia natural de S. variegatoides recurriendo a metodologías usadas y eventualmente validar criterios aplicados para las otras especies, por ejemplo, para clasificar individuos según su edad y así disponer de un mejor conocimiento de la estructura poblacional para esta especie.

Dado que esta especie es considerada dañina para diferentes cultivos agrícolas y especies forestales (Monge, 2009), la información sobre su ciclo reproductivo es útil, dado que la natalidad representa un parámetro importante en la dinámica poblacional de las especies. En este sentido, en los casos en que se requiera intervenciones para mantener las poblaciones en un nivel inferior a que alcance la condición de plaga, conviene hacerlas previo a la época reproductiva, de tal manera que sea menor la cantidad de individuos afectados.

\section{AGRADECIMIENTOS}

Agradezco a la familia Shutt Valle por las facilidades para realizar este estudio, así como a Luko Hilje por la guía en la investigación. A los revisores anónimos por sus sugerencias al manuscrito.

\section{REFERENCIAS}

Best, T.L. (1995). Sciurus variegatoides. Mammalian Species, 500, 1-6. DOI: $10.2307 / 3504262$

Birnes, E.C., Bowles, J.B., Timm, R.M., \& Williams, S.L. (1974). Mammalian distributional records in Yucatán and Quintana Roo, with comments on reproduction, structure, and status of peninsular populations. Bell Museum of Natural History, University of Minnesota, Occasional Papers, 13, 1-12.

Boucher, D.H. (1981). Seed predation by mammals and forest dominance by Quercus oleoides, a tropical lowland oak. Oecologia, 49, 409-414. DOI: 10.1007/BF00347608

Brown, L.M., \& McGuire, R.J. (1975). Field ecology of the exotic Mexican red-bellied squirrel in Florida. Journal of Mammalogy, 56, 405-419. DOI: 10.2307/1379370 
Fleming, T.H. (1970). Notes on the rodent faunas of two Panamanian forests. Journal of Mammalogy, 51, 473490. DOI: $10.2307 / 1378388$

Giacalone, J., Glanz, W.E., \& Leigh, E.G. (1990). Adición: Fluctuaciones poblacionales a largo plazo de Sciurus granatensis en relación con la disponibilidad de frutos. En A. S. Rand, D. M. Windsor, \& E. G. Leigh (Eds.), Ecología de un bosque tropical: ciclos estacionales y cambios a largo plazo (pp. 331-335). Panamá: Smithsonian Tropical Research Institute.

Glanz, W.E. (1984). Food and habitat use by two sympatric Sciurus species in Panama. Journal of Mammalogy, 65, 342-347. DOI: $10.2307 / 1381179$

Glanz, W.E., Thorington, R.W., Giacalone-Madden, J., \& Heaney, L.R. (1990). Utilización estacional de alimentos y tendencias demográficas de Sciurus granatensis. En A. S. Rand, D. M. Windsor, \& E. G. Leigh (Eds.), Ecología de un bosque tropical: ciclos estacionales y cambios a largo plazo (pp. 317-3309). Panamá: Smithsonian Tropical Research Institute.

Goodwin, G.G. (1946). Mammals of Costa Rica. Bulletin of the American Museum of Natural History, 87, 275- 473.

Harris, W.P. (1937). Revision of Sciurus variegatoides, a species of Central American squirrel. Museum of Zoology University of Michigan. Miscellaneous publications, 38, 5-39.

Hayssen, V. (2008). Patterns of body and tail length and body mass in Sciuridae Journal of Mammalogy, 89, 852-873.

Heaney, L.R. (1991). Sciurus granatensis (Ardilla, Ardilla Roja, Chisa, Red-tailed Squirrel). En D. Janzen (Ed.), Historia Natural de Costa Rica (pp. 503-504). San José, Costa Rica: Organización de Estudios Tropicales y Editorial de la Universidad de Costa Rica.

Heaney, L.R., \& Thorington, R.W. (1978). Ecology of Neotropical red-tailed squirrels, Sciurus granatensis, in the Panama Canal Zone. Journal of Mammalogy, 59, 846-851. DOI: $10.2307 / 1380149$

Henn, J.J., McCoy, M.B., \& Vaughan, C.S. (2014). Beach almond (Terminalia catappa, Combretaceae) seed production and predation by scarlet macaws (Ara macao) and variegated squirrels (Sciurus variegatoides). Revista Biología Tropical, 62, 929-938. DOI: 10.15517/rbt.v62i3.14060

Hilje, L., \& Monge, J. (1988). Lista preliminar y consideraciones generales acerca de los animales vertebrados plaga en Costa Rica. Manejo Integrado de Plagas, 10, 39-52.

Janzen, D.H. (1971). Escape of juvenile Dioclea megacarpa (Leguminosae) vines from predators in deciduous tropical forest. The American Naturalist, 105, 97-112. DOI: $10.1086 / 282709$

Janzen, D.H. (1972). Escape in space by Sterculia apetala seeds from the bug Dysdercus fasciatus in a Costa Rican deciduous forest.Ecology, 53, 350-361.DOI: 10.2307/1934092

Janzen, D.H. (1982a). Natural history of guacimo fruits (Sterculiaceae: Guazuma ulmifolia) with respect to consumption by large mammals. American Journal of Botany, 69, 1240-1250. DOI: 10.1002/j.1537-2197.1982. tb13369.x
Janzen, D.H. (1982b). Fruit traits, and seed consumption by rodents, of Crecentia alata (Bignoniaceae) in Santa Rosa National Park, Costa Rica. American Journal of Botany, 69, 1258-1268. DOI: 10.1002/j.1537-2197.1982.tb13371.x

Jones, J.K., Genoways, H.H., \& Lawlor, T.E. (1974). Annotated checklist of mammals of the Yucatan Peninsula, Mexico ii. Rodentia. Occasional Papers the Museum Texas Tech University, 22, 1-24.

McPherson, A.B. (1971). A new subspecies of the squirrel Sciurus variegatoides Ogilby from Costa Rica. Revista Biología Tropical, 19, 191-194.

McPherson, A.B. (1985). A biogeographical analysis of factors influencing the distribution of Costa Rican rodents. Brenesia, 23, 97-273.

Méndez, E. (1993). Roedores de Panamá. Panamá: Impresora Pacífico.

Monge, J. (2009). Roedores plaga de América Central. San José, Costa Rica: Editorial de la Universidad de Costa Rica.

Monge, J., \& Hilje, L. (2006). Hábitos alimenticios de la ardilla Sciurus variegatoides (Rodentia: Sciuridae) en la Península de Nicoya, Costa Rica. Revista Biología Tropical, 54, 681-686. DOI: 10.15517/rbt.v54i2.14113

Ramos-Lara, N., \& Cervantes, F. A. (2011). Ecology of the Mexican red-bellied squirrel (Sciurus aureogaster) in Michoacan, Mexico. The Southwestern Naturalist, 56, 400-403. DOI: 10.1894/N02-RTS-11.1

Reid, F.A. (2009). A Field Guide to the Mammals of Central America and Southeast Mexico. New York, USA: Oxford University Press.

Rose, L.M.,Perry, S., Panger, M.A.,Jack, K., Manson,J.H., Gros-Louis, J., ... Vogel, E. (2003). Interspecific interactions between Cebus capucinus and other species: Data from three Costa Rican sites. International Journal of Primatology, 24, 759-796. DOI: 10.1023/A:1024624721363

Schemske, D.W. (1980). Floral ecology and hummingbird pollination of Combretum farinosum in Costa Rica. Biotropica, 12, 169-181. DOI: $10.2307 / 2387968$

Somarriba, E., Trivelato, M., Villalobos, M., Suárez, A., Benavides, P., Morán, K., ... López, A. (2003). Diagnóstico agroforestal de pequeñas fincas cacaoteras orgánicas de indígenas Bribri y Cabécar de Talamanca, Costa Rica. Agroforestería de la Américas, 10, 24-30.

Thorington, R.W., \& Hoffmann, R.S. (2005). Family Sciuridae. En D. E. Wilson, \& D. M. Reeder (Eds.), Mammal species of the world: a taxonomic and geographic reference (Vol.1, $3^{\text {rd }}$ ed.) (pp. 754-818). Baltimore, Maryland: Johns Hopkins University Press.

Thorington, R.M., Koprowski, J.L., Steele, M.A., \& Whatton, J.F. (2012). Squirrels of the world. Baltimore, Maryland: The Johns Hopkins University Press.

Tosi, J. (1969). Mapa ecológico de Costa Rica. Escala 1:750 000. San José, Costa Rica: Centro Científico Tropical. 